\title{
Symptomatic dengue infection during pregnancy and the risk of stillbirth in Brazil, 2006-12: a matched case-control study
}

\author{
Enny S Paixão, Maria da Conceição N Costa, Maria Glória Teixeira, Katie Harron, Marcia Furquim de Almeida, Mauricio L Barreto, \\ Laura C Rodrigues
}

\begin{abstract}
Summary
Background Maternal infections during pregnancy can increase the risk of fetal death. Dengue infection is common, but little is known about its role in fetal mortality. We aimed to investigate the association between symptomatic dengue infection during pregnancy and fetal death.
\end{abstract}

Methods We did a nested case-control study using obstetrician-collected data from the Brazilian livebirth information system (SINASC), the mortality information system (SIM), and the national reportable disease information system (SINAN). We identified all pregnancies ending in stillbirth and a random sample of livebirths between Jan 1, 2006, and Dec 31, 2012. We did linkage to determine which mothers were diagnosed with dengue infection during pregnancy. By use of stillbirths as cases and a sample of matched livebirths as a control, we calculated matched odds ratios (mORs) using conditional logistic regression adjusted for maternal age and education.

Findings $275(0 \cdot 2 \%)$ of 162188 women who had stillbirths and $1507(0 \cdot 1 \%)$ of 1586105 women who had livebirths were diagnosed with dengue infection during pregnancy. Symptomatic dengue infection during pregnancy almost doubled the odds of fetal death (mOR 1.9, 95\% CI 1.6-2.2). The increase in risk was similar when analyses were restricted to laboratory-confirmed cases of dengue infection $(1 \cdot 8,1 \cdot 4-2 \cdot 4)$. Severe dengue infection increased the risk of fetal death by about five times $(4 \cdot 9,2 \cdot 3-10 \cdot 2)$.

Interpretation Symptomatic dengue infection during pregnancy is associated with an increased risk of fetal death. We recommend further epidemiological and biological studies of the association between dengue and poor birth outcomes to measure the burden of subclinical infections and elucidate pathological mechanisms.

Funding Brazilian National Council for Scientific and Technological Development, Horizon 2020.

\section{Introduction}

Fetal death is a common adverse outcome of pregnancy, even in high-income countries. Although the global burden of stillbirths declined by $25 \cdot 5 \%$ between 2000 and 2015, there were still 2.6 million stillbirths worldwide in 2015. ${ }^{1}$ The incidence of stillbirth varies from an estimated four in 1000 births in high-income countries to 26 in 1000 births in low-income and middleincome countries. ${ }^{1}$

Infection during pregnancy is believed to cause $10-25 \%$ of all fetal deaths in high-income countries, ${ }^{2}$ with infection in early pregnancy carrying an increased risk; ${ }^{3,4}$ infection during the first 28 weeks of gestation causes $15 \%$ of all fetal deaths, while infection after 28 weeks of gestation causes $6 \%$ of all fetal deaths. ${ }^{5}$ Viral infections are estimated to contribute to $14.5 \%$ of all fetal deaths, ${ }^{6}$ although some unexplained stillbirths could be due to undiagnosed viral infections. ${ }^{\text {? }}$

The confirmation of Zika virus infection as a cause of congenital anomalies and stillbirth highlighted the importance of original research on the effect of flaviviruses on stillbirths. ${ }^{8}$ A review by McClure and colleagues $^{9}$ in 2014 mentioned dengue infection in pregnant women as a cause of fetal death, yet no population-based studies showing this association have been done. In 2010, a systematic review ${ }^{10}$ concluded that it was unclear whether dengue infection during pregnancy had any negative effect on fetal outcomes. Another systematic review ${ }^{11}$ found that only one study ${ }^{12}$ of dengue infection during pregnancy and fetal death had a comparison group. That study ${ }^{12}$ included 13 stillbirths (nine of which had been exposed to dengue virus), and the crude relative risk of stillbirth was 6.7 (95\% CI 2.1-21.3) in women with symptomatic dengue infection compared with women without. All other evidence to date comes from case reports or case series, none of which have included appropriate controlling for confounding variables or population control groups.

The mechanisms whereby dengue infection could lead to stillbirth are unknown, but three main hypotheses exist: the symptoms of dengue infection in the mother have a direct effect on the fetus (severe dengue); dengue infection causes changes that affect the placenta; and the dengue virus has a direct effect on the fetus. ${ }^{2}$ The incidence of dengue infection is increasing, and about half of the global population is at risk, including women of reproductive age, ${ }^{13}$ however, the effect of dengue infection during pregnancy on fetal mortality remains unclear. Therefore, we investigated the association between stillbirth and symptomatic dengue infection during pregnancy.

\author{
Lancet Infect Dis 2017; \\ 17: 957-64 \\ See Comment page 886 \\ London School of Hygiene \& \\ Tropical Medicine, London, UK \\ (E S Paixão MSc, K Harron PhD, \\ Prof L ( Rodrigues PhD); \\ Instituto de Saúde Coletiva \\ Salvador, Bahia \\ Brazil (E S Paixão, \\ Prof M dC N Costa PhD \\ Prof M G Teixeira PhD); \\ Center of Data and Knowledge \\ Integration for Health \\ (CIDACS), Instituto Gonçalo \\ Moniz, Salvador, Bahia, Brazil \\ (E S Paixão, Prof M G Teixeira, \\ Prof M L Barreto PhD, \\ Prof L ( Rodrigues); and \\ Universidade de São Paulo, \\ São Paulo, Brazil \\ (Prof M F de Almeida PhD, \\ Prof M L Barreto) \\ Correspondence to: \\ Enny S Paixão, London School of \\ Hygiene \& Tropical Medicine, \\ London WC1E 7HT, UK \\ enny.cruz@Ishtm.ac.uk
}


Research in context

\section{Evidence before this study}

Dengue is endemic in many regions of the world, including the Americas, Asia, and Oceania. A better understanding of the effect of dengue infection during pregnancy is needed to elucidate the burden of this disease, including the economic consequences, and to inform initiatives to reduce fetal and neonatal mortality. Although the causes of stillbirth are not completely understood, infections during pregnancy contribute to a proportion of stillbirths worldwide, and strong evidence exists for a role of some infections, such as syphilis, toxoplasmosis, cytomegalovirus, and parvovirus B19. However, little is known about the role of dengue infection in fetal mortality. Only one study with a comparison group (hospital-based) has previously explored the evidence, showing a higher risk of stillbirth among pregnancies with symptomatic dengue than among those without.

\section{Added value of this study}

To our knowledge, this study is the first to quantify the risk of stillbirth among women with symptomatic dengue infection during pregnancy using a population-based approach, with a sufficiently large sample size and controlling for confounders. We showed that symptomatic dengue infection during pregnancy roughly doubled the risk of stillbirth and that the effect was increased during the acute phase and for severe dengue.

\section{Implications of all the available evidence}

If the association between dengue and stillbirth were to be shown in other studies, recommendations should be made for close monitoring of pregnant women with dengue symptoms and their inclusion as an at-risk population in dengue control programmes, to strengthen actions for individual protection of pregnant women.

\section{Methods}

\section{Study design}

We did a population-based nested case-control study with linkage of routine data for all pregnant women whose outcomes were livebirth or stillbirth in Brazil from Jan 1, 2006, to Dec 31, 2012. Data were extracted from three Brazilian databases: the Brazilian livebirth information system (SINASC), the mortality information system (SIM), and the national reportable disease information system (SINAN). SINASC contains records of all livebirths in Brazil, SIM contains records of all stillbirths in Brazil, and SINAN contains records of all notifiable diseases in Brazil.

SINASC consists of data from livebirth notifications, which is a legal document completed by the health professional who assisted the delivery. This document includes information about the mother (name, place of residence, age, marital status, education, whether she had a stillbirth or a child who died), the pregnancy (length of gestation, type of delivery), and the newborn baby (birthweight, presence of congenital anomalies). ${ }^{14}$ The completeness of the data from SINASC is very high, with most variables completed in more than $90 \%$ of cases; the exception being information about whether the mother had a previous stillbirth or abortion. ${ }^{15}$

Data in SIM come from the death certificate, which is also a legal document. ${ }^{16}$ The proportion of records with missing data varies by variable and is around $20 \%$ for maternal education and number of previous fetal deaths or abortions. Data about notifiable diseases, including personal information about the patient (name, place of residence, age, education), symptoms of disease, laboratory tests, and disease severity, are captured in SINAN, which contained reasonably complete data for the variables we used for linkage and in the final analyses $(<0.05 \%$ of records were excluded because of missing name). SINAN contains two sources of information about maternal age (age and date of birth). About $5 \%$ of dengue cases did not have a final classification of severity. Laboratory confirmation was not required for notification of dengue cases because dengue was the main (and for some years the only) vector-borne disease circulating in Brazil (yellow fever and malaria occur in restricted areas; notification of Zika and chikungunya viruses was not introduced until 2014).

This study was approved by the Federal University of Bahia (protocol number 26797814.7.0000.5030) and the London School of Hygiene \& Tropical Medicine (ethics reference number 10269).

\section{Procedures}

In SIM, stillbirth was defined as the death of a product of conception before the expulsion or complete extraction from the body of the pregnant woman, occurring from 22 weeks or weighing more than $500 \mathrm{~g}$, according to the Brazilian definition. ${ }^{16}$ In SINASC, livebirth was defined as a product of conception that, after separation from the mother's body (independent of the duration of pregnancy), breathes or shows any other signs of life, such as a heartbeat, umbilical cord pulsation, or definite movement of voluntary muscles, according to the Brazilian definition. ${ }^{14}$

Records with missing or implausible names for the mother, multiple pregnancies, congenital anomalies, birth or stillbirth from municipalities without dengue infection notification, and an uncertain links status were excluded from case and control groups. Multiple pregnancies and congenital anomalies were excluded because they might be causes or related to causes of stillbirth.

Cases were stillbirths recorded in SIM. As controls, we used a random sample of ten livebirths (without replacement), matched to cases by month and calendar year of birth, and municipality of residence. We randomly ordered cases and controls within month and year and 
place of birth groups, and selected the first case in the group to be matched to the first ten controls in the group. The second case was matched to controls 11-20 and so on, forming pairwise combinations. We selected ten controls per case to enable us to control for potential confounders, such as dengue seasonality, intensity of virus circulation, and social environment.

The exposure in this study was a notified confirmed case of dengue infection during pregnancy that resulted in the birth of a case or control. Dengue in Brazil can be confirmed on the basis of clinical epidemiological criteria, including the presence of clinical symptoms of dengue in the same area and time as other confirmed cases of dengue, or on the basis of clinical laboratory criteria, including the presence of clinical symptoms and laboratory confirmation (a positive result from one of IgM detection by ELISA, viral RNA detection by PCR, NS1 viral antigen detection, or positive viral culture).

We considered a fetus to be exposed if their mother was notified to SINAN and confirmed as a dengue case (and therefore their records were linked to a notification of dengue during the pregnancy). Dengue infection during pregnancy refers to all confirmed cases of dengue (based on clinical epidemiological and clinical laboratory data); dengue infection during pregnancy, laboratory confirmed refers to only the laboratory-confirmed cases. We classified dengue into two clinical categories: dengue fever as a self-limiting disease (fever with severe headache, pain behind the eyes, muscle and joint pain, and rash) or severe dengue. We classified severe dengue as all cases of dengue that the Brazilian Ministry of Health classified as dengue haemorrhagic fever, dengue shock syndrome, or complicated dengue. Complicated dengue is a Brazilian definition for all severe cases of dengue that do not meet the WHO criteria for dengue haemorrhagic fever (fever, haemorrhagic evidence, thrombocytopenia, and evidence of plasma leakage) and cannot be classified as mild selflimited disease because of their severity. The term complicated dengue was used when a probable case of dengue presented with severe changes in the nervous system, cardiorespiratory dysfunction, insufficient hepatic function, gastrointestinal bleeding, cavity spills, thrombocytopenia of 50000 platelets per $\mu \mathrm{L}$ or fewer, or leucometry 1000 cells per $\mu \mathrm{L}$ or fewer. ${ }^{17}$ We used only two clinical categories because of the small number of observations and because dengue haemorrhagic fever and dengue shock syndrome are rare.

\section{Linkage procedure}

SINAN dengue records were linked probabilistically with the cohort of pregnant women from SINASC and SIM to identify those who had dengue infection during pregnancy. Name, age, and place of residence of the mother at the time of delivery or diagnosis were used in the matching process. We excluded records with missing or implausible names and duplicates. Match weight calculations were based on the Fellegi-Sunter method. ${ }^{18}$

We created a gold-standard dataset using all stillbirth records for 2 years of data (2009 and 2010) and all livebirth records within two states (Ceará and Espirito

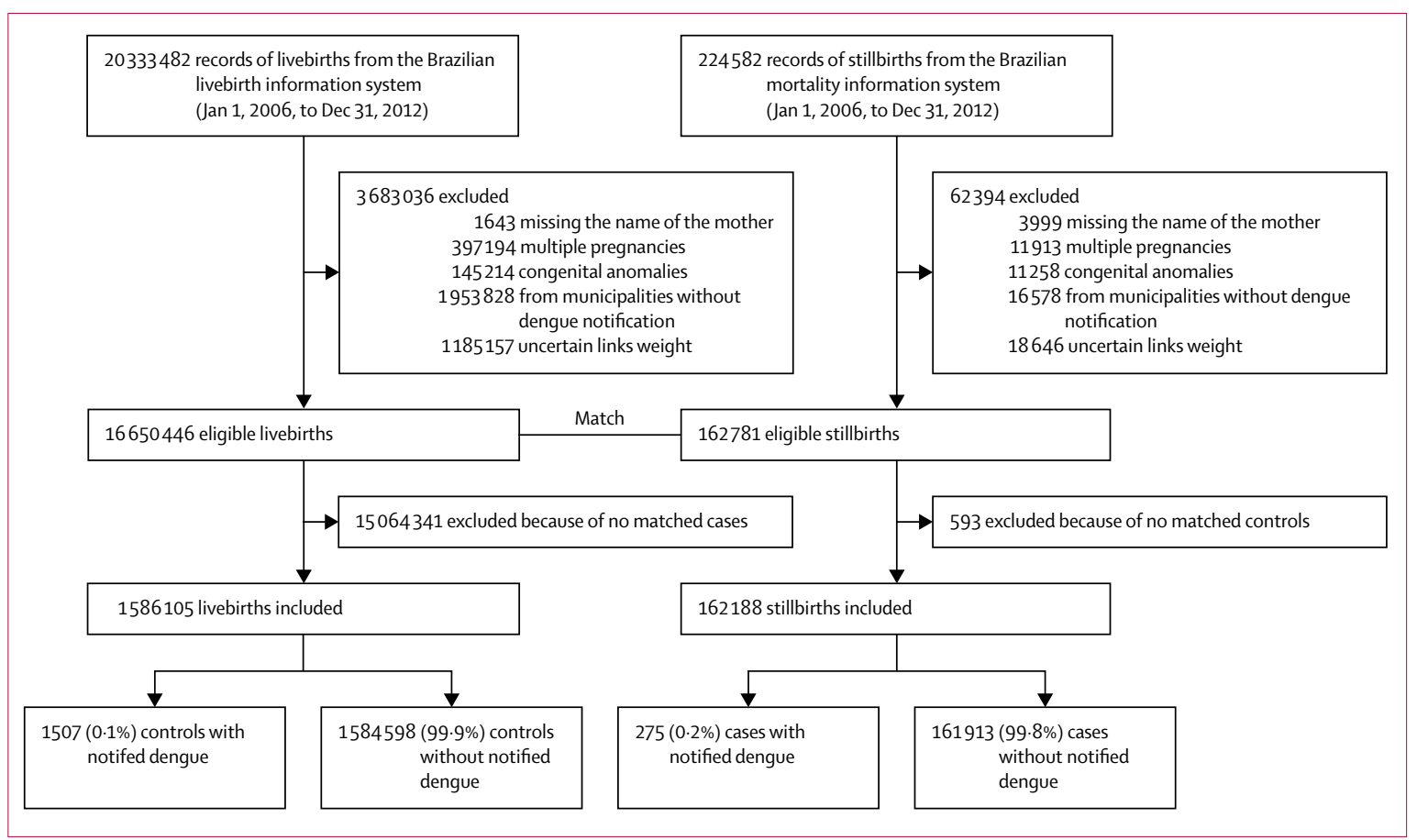

Figure 1: Study profile

One case was matched to ten controls by month and calendar year and municipality of the mother. 


\begin{tabular}{|c|c|c|}
\hline & Cases $(n=162188)$ & $\begin{array}{l}\text { Controls } \\
(n=1586105)\end{array}$ \\
\hline \multicolumn{3}{|l|}{ Age of the mother } \\
\hline$<20$ years & $35752(25 \cdot 7 \%)$ & $429374(27 \cdot 0 \%)$ \\
\hline $20-35$ years & $85534(61 \cdot 5 \%)$ & $1038176(65 \cdot 5 \%)$ \\
\hline$>35$ years & $17715(12 \cdot 8 \%)$ & $118511(7 \cdot 5 \%)$ \\
\hline Missing & $23187(14 \cdot 3 \%)$ & $44(0 \cdot 2 \%)$ \\
\hline \multicolumn{3}{|l|}{ Maternal education } \\
\hline None & $9235(7 \cdot 9 \%)$ & $25596(1 \cdot 7 \%)$ \\
\hline $1-3$ years & $15105(12 \cdot 8 \%)$ & $120385(7 \cdot 8 \%)$ \\
\hline $4-7$ years & $38365(32 \cdot 6 \%)$ & $456186(29 \cdot 4 \%)$ \\
\hline $8-11$ years & $42393(36 \cdot 1 \%)$ & $715653(46 \cdot 2 \%)$ \\
\hline$>11$ years & $12457(10 \cdot 6 \%)$ & 231448 (14.9\%) \\
\hline Missing & $44633(27 \cdot 5 \%)$ & $36837(2 \cdot 3 \%)$ \\
\hline \multicolumn{3}{|l|}{ Gestational age } \\
\hline$<22$ weeks & $9862(6.6 \%)$ & $904(0.1 \%)$ \\
\hline 22-27 weeks & $33309(22 \cdot 4 \%)$ & $5758(0 \cdot 4 \%)$ \\
\hline 28-31 weeks & $24696(16 \cdot 6 \%)$ & $10627(0 \cdot 7 \%)$ \\
\hline 32-36 weeks & $38592(25 \cdot 9 \%)$ & $94795(6 \cdot 1 \%)$ \\
\hline 37-41 weeks & $40501(27 \cdot 2 \%)$ & $1414917(91 \cdot 0 \%)$ \\
\hline$>42$ weeks & $1987(1 \cdot 3 \%)$ & $26309(1 \cdot 7 \%)$ \\
\hline Missing & $13241(8 \cdot 2 \%)$ & $32795(2 \cdot 1 \%)$ \\
\hline \multicolumn{3}{|c|}{ Previous fetal death or abortion } \\
\hline No & $48337(41 \cdot 5 \%)$ & $1163696(87 \cdot 5 \%)$ \\
\hline Yes & $68012(58 \cdot 5 \%)$ & $166696(12 \cdot 5 \%)$ \\
\hline Missing & $45839(28 \cdot 3 \%)$ & $255713(16 \cdot 1 \%)$ \\
\hline \multicolumn{3}{|c|}{ Birth or death weight } \\
\hline$\geq 2500 \mathrm{~g}$ & $44245(30 \cdot 4 \%)$ & $1467719(92 \cdot 8 \%)$ \\
\hline $1500-2500 \mathrm{~g}$ & $36285(24 \cdot 9 \%)$ & $97334(6 \cdot 1 \%)$ \\
\hline$<1500 \mathrm{~g}$ & $65081(44 \cdot 7 \%)$ & $17214(1 \cdot 1 \%)$ \\
\hline Missing & $16577(10 \cdot 2 \%)$ & $3838(0.2 \%)$ \\
\hline \multicolumn{3}{|c|}{ Dengue during pregnancy } \\
\hline No & $161913(99 \cdot 8 \%)$ & $1584598(99 \cdot 9 \%)$ \\
\hline $\begin{array}{l}\text { Dengue with lab } \\
\text { confirmation }\end{array}$ & $95(0.06 \%)$ & $554(0.03 \%)$ \\
\hline $\begin{array}{l}\text { Dengue without lab } \\
\text { confirmation }\end{array}$ & $180(0 \cdot 14 \%)$ & $953(0.07 \%)$ \\
\hline
\end{tabular}

Data are n (\%). \% for all categories other than "Missing" were calculated with exclusion of those with missing data from the denominator; \% for "Missing" rows used total cases in the denominator.

Table 1: Maternal characteristics for 162188 stillbirths and 1586105 live singleton births in Brazil for 2006-12

Santo) for 1 year (2010) of data. We did a manual review of record pairs to identify true matches (records belonging to the same mother) and estimate m-probabilities (P[agreement $\mid$ match]), which is the probability of a match given agreement on each identifier. To calculate u-probabilities (P[agreement|non-match]), we used a subset of SINAN to create a set of non-matches by crossjoining all record pairs and removing record pairs belonging to the same individual. Record pairs were then ordered by match weight and manually inspected to identify obvious non-links that had high weights and probable links with low weights. Only links and non-links with a high degree of certainty were retained for the casecontrol study to avoid misclassification; uncertain links (when we could not determine whether or not a woman had dengue infection) were excluded from the analyses.

To evaluate the quality of the linkage algorithm, we compared the links obtained using the probabilistic algorithm with the gold standard created by manual review. This validation showed that sensitivity was $64 \%$ for stillbirths and $62 \%$ for livebirths, positive predictive value was $94 \%$ for stillbirths and $95 \%$ for livebirths, and specificity was more than $99 \%$ for both. The missed matches occurred randomly in cases and controls (appendix). The procedures and evaluation of the matching process are the subject of a separate paper (unpublished).

\section{Statistical analysis}

To calculate the sample size, we did two preliminary deterministic linkages of records with $100 \%$ agreement in the three identifiers (name, age, and municipality of the mother): the first between SINAN and SIM, to calculate the minimum proportion of mothers of stillbirths who had dengue infection during pregnancy $(0 \cdot 05 \%)$, and the second between SINAN and SINASC, to calculate the minimum proportion of mothers of livebirths who had dengue infection during pregnancy $(0.03 \%)$. We estimated that, with 94755 cases, we would be able to detect a matched odds ratio (mOR) of 1.6 using the parameters proportion exposed $(0.05 \%$ for stillbirths and $0.03 \%$ for livebirths), $80 \%$ power, $95 \%$ confidence level, and a ratio of one case to ten controls (appendix).

For the overall association, we estimated crude mORs with univariate conditional logistic regression and adjusted mORs with conditional logistic regression, controlling for maternal age and maternal education (as a proxy for socioeconomic status). For a sensitivity analysis of the validity of dengue clinical diagnosis, we repeated the analysis with laboratory-confirmed dengue cases only. We investigated the effect of dengue by disease severity and time between the disease and outcome. We did an analysis stratified by report of a previous fetal death or abortion to explore whether dengue infection had a different effect in pregnancies with a high risk of fetal death. To investigate the potential effect of missing data, we did another sensitivity analysis in which we assumed that all missing data for confounding variables in the cases were in the low-risk groups and all missing data for the controls were in the high-risk groups.

The population attributable fraction (PAF; $\mathrm{PAF}=[\mathrm{p} 1(\mathrm{OR}-1)] / \mathrm{OR})$ was calculated with the punafcc package in Stata, which uses a logistic regression method ${ }^{19}$ and provides PAF (and 95\% CIs) with adjustment for confounding variables by combining adjusted ORs and the observed incidence of dengue among cases (stillbirths). We used Stata version 14.1 software for the statistical analyses. 


\section{Role of the funding source}

The funder of this study had no role in study design, data collection, data analysis, interpretation, or writing of the report. All authors had full access to all de-identified data in the study and had final responsibility for the decision to submit for publication.

\section{Results}

SIM recorded 224582 stillbirths and SINASC recorded 20333482 livebirths during the study period. After exclusions, 162781 stillbirths were eligible for the study. No matched control was found for $593(0 \cdot 4 \%)$ stillbirths and so these were excluded. The final study population included 162188 stillbirths and 1586105 livebirths, with 275 stillbirths and 1507 livebirths exposed to dengue (figure 1).

The characteristics of cases and controls are shown in table 1 . The proportion of missing values for all variables was higher among cases than among controls, including values for classic risk factors for stillbirth, such as previous fetal death or abortion (28.3\% vs $16 \cdot 1 \%)$ and maternal education $(27.5 \%$ vs $2 \cdot 3 \%)$. This finding was also true for age, birth or death weight, and gestational age.

The risk of stillbirth among all births recorded in the information systems was 11 in 1000 livebirths. The risk of stillbirth in women with dengue infection during pregnancy was 15 (95\% CI 13-17) in 1000 pregnancies. Overall, dengue infection was laboratory confirmed in more than $30 \%$ of the dengue cases recorded. Dengue infection during pregnancy was more common in mothers of cases $(0.2 \%)$ than in mothers of controls $(0 \cdot 1 \%$; table 1$)$. Potential confounding variables (ie, variables associated with dengue infection and stillbirth) were maternal education $(\mathrm{p}<0.001)$ and previous fetal death or abortion ( $\mathrm{p}=0.02$; table 2 ).

The crude association between symptomatic dengue infection during pregnancy and stillbirth was mOR 1.8 (95\% CI 1.6-2.0), and was similar when adjusted for maternal education and maternal age (mOR 1.9, 95\% CI 1.6-2.2). The analysis restricted to laboratoryconfirmed dengue gave a similar adjusted mOR $(1.8$, 1.4-2.4). We investigated whether the association between dengue infection and stillbirth depended on previous fetal death or abortion, but the mOR did not differ according to this variable (table 3). The risk of fetal death was dependent on the time between the first symptoms of dengue infection and the date of livebirth or stillbirth; the risk appeared to be bimodal. The risk of fetal death peaked in the first 20 days after the onset of symptoms $(4 \cdot 9,3 \cdot 2-7 \cdot 5$; to a greater extent when dengue was severe; table 3 and figure 2). After 10 days, the risk of mortality remained increased $(1 \cdot 7,1 \cdot 4-2 \cdot 0)$, but was roughly constant until the end of pregnancy (figure 2 ). Severe dengue during pregnancy increased the risk of stillbirth about five times $(4 \cdot 9,2 \cdot 3-10 \cdot 2)$, almost three times that of mild dengue $(1 \cdot 7,1 \cdot 5-2 \cdot 0$; table 3$)$.

A sensitivity analysis was done because the proportion of missing data was higher among cases than among

\begin{tabular}{|c|c|c|c|}
\hline & $\begin{array}{l}\text { With notified } \\
\text { dengue }(n=1507)\end{array}$ & $\begin{array}{l}\text { Without notified dengue } \\
(\mathrm{n}=1584598)\end{array}$ & $p$ value \\
\hline Age of the mother & & & $p=0.76$ \\
\hline$<20$ years & $392(26 \cdot 0 \%)$ & 428982 (27.1\%) & \\
\hline $20-35$ years & $1006(66 \cdot 8 \%)$ & $1037170(65 \cdot 5 \%)$ & \\
\hline$>35$ years & $109(7 \cdot 2 \%)$ & $118402(7 \cdot 5 \%)$ & \\
\hline Missing & $0(0 \%)$ & $44(0.0 \%)$ & \\
\hline Maternal education & & & $p<0.001$ \\
\hline None & $11(0.8 \%)$ & $25585(1 \cdot 7 \%)$ & \\
\hline $1-3$ years & $73(4 \cdot 9 \%)$ & $120312(7 \cdot 8 \%)$ & \\
\hline $4-7$ years & $431(29 \cdot 2 \%)$ & $455755(29 \cdot 4 \%)$ & \\
\hline 8-11years & $763(51 \cdot 7 \%)$ & $714890(46 \cdot 2 \%)$ & \\
\hline$>11$ years & $197(13 \cdot 4 \%)$ & 231251 (14.9\%) & \\
\hline Missing & $32(2 \cdot 1 \%)$ & $36805(2 \cdot 3 \%)$ & \\
\hline Gestational age & & & $p=1.53$ \\
\hline$<22$ weeks & $1(0.1 \%)$ & $903(0.1 \%)$ & \\
\hline 22-27 weeks & $2(0.1 \%)$ & $5756(0 \cdot 4 \%)$ & \\
\hline 28-31 weeks & $7(0.5 \%)$ & $10620(0.7 \%)$ & \\
\hline 32-36 weeks & $89(6.0 \%)$ & 94706 (6.0\%) & \\
\hline 37-41 weeks & $1369(92 \cdot 0 \%)$ & $1413548(91 \cdot 1 \%)$ & \\
\hline$>42$ weeks & $20(1 \cdot 3 \%)$ & $26289(1 \cdot 7 \%)$ & \\
\hline Missing & $19(1 \cdot 3 \%)$ & $32776(2 \cdot 1 \%)$ & \\
\hline Previous fetal death or abortion & & & $p=0.02$ \\
\hline No & $1102(85 \cdot 4 \%)$ & $1162594(87 \cdot 5 \%)$ & \\
\hline Yes & $188(14 \cdot 6 \%)$ & $166508(12 \cdot 5 \%)$ & \\
\hline Missing & $217(14 \cdot 4 \%)$ & $255496(16 \cdot 1 \%)$ & \\
\hline Birth or death weight & & & $p=0.59$ \\
\hline$\geq 2500 \mathrm{~g}$ & $1400(93 \cdot 1 \%)$ & $1466319(92 \cdot 8 \%)$ & \\
\hline $1500-2500 \mathrm{~g}$ & $93(6 \cdot 2 \%)$ & $97241(6 \cdot 1 \%)$ & \\
\hline$<1500 \mathrm{~g}$ & $11(0.7 \%)$ & $17203(1 \cdot 1 \%)$ & \\
\hline Missing & $3(0.2 \%)$ & $3835(0.2 \%)$ & \\
\hline
\end{tabular}

Data are n (\%). \% for all categories other than "Missing" were calculated with exclusion of those with missing data from the denominator; \% for "Missing" rows used total cases in the denominator.

Table 2: Maternal characteristics for 1586105 live singleton births in Brazil by dengue status

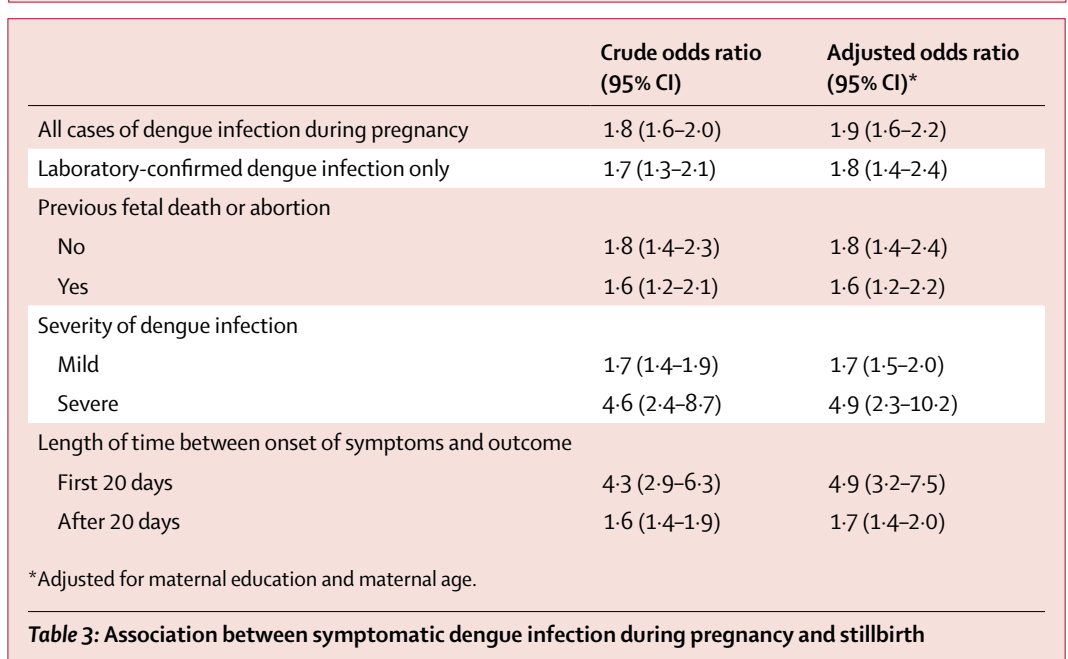

controls. We assumed that all missing data for confounding variables in the cases were in the low-risk groups and that all missing data in the controls were in 

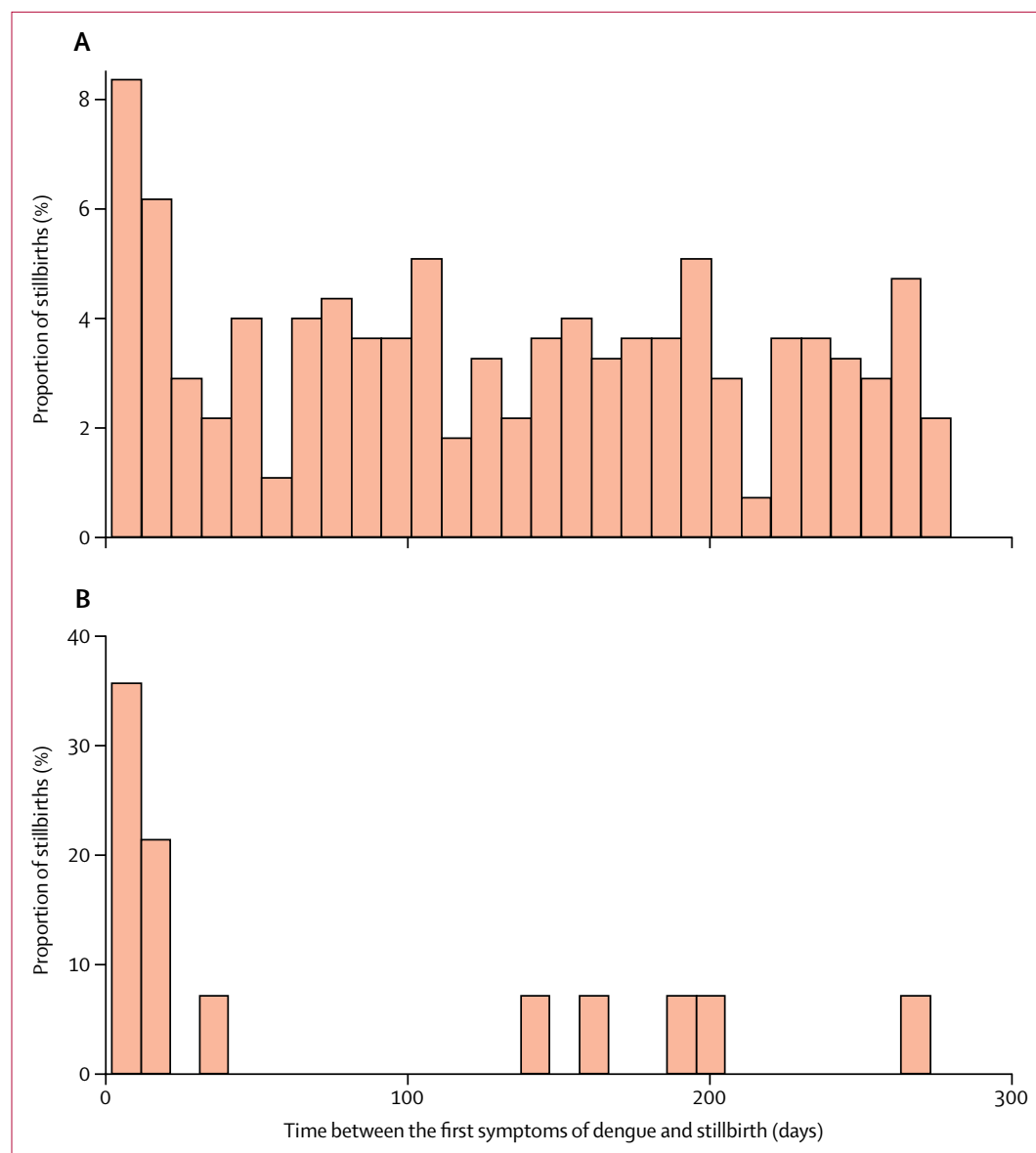

Figure 2: Time between the first symptoms of dengue and stillbirth delivery

(A) All dengue cases. (B) Severe dengue cases only.

the high-risk groups. The magnitude of the association was very similar (mOR $1 \cdot 8,95 \%$ CI $1 \cdot 5-2 \cdot 0)$.

The prevalence of symptomatic dengue infection during pregnancy was low $(0 \cdot 1 \%$ of mothers of controls were notified of dengue infection during pregnancy), so the PAF was also low; $0.08 \%$ of all stillbirths during the period were attributed to dengue infection, with marked yearly variation and a maximum of $0.13 \%$ during epidemic years (appendix).

\section{Discussion}

We found that symptomatic dengue infection during pregnancy roughly doubled the risk of stillbirth, with the highest risk observed in the first 20 days after disease onset and in women with severe disease. The increase in risk was observed for all cases of dengue (either based on clinical epidemiological data or on clinical laboratory data) and when the analysis was restricted to only laboratory-confirmed dengue. Dengue infection during pregnancy is reasonably rare, and the proportion of all fetal deaths attributable to dengue during pregnancy in Brazil during the period was relatively small, but was larger during epidemic years.
To our knowledge, this is the first study to investigate the risk of stillbirth among women with dengue infection during pregnancy by use of a populationbased approach, with a sufficiently large sample size and controlling for confounders. However, we were not able to adjust for all possible confounding factors because we were dependent on routine information obtained from notification system records. The results of our study were consistent with those of a study done in French Guiana, ${ }^{12}$ which found that the risk of stillbirth was increased in pregnancies with symptomatic dengue. Stillbirth has been associated with other viral maternal infections and the magnitude of this association varies depending on the virus involved, the severity of maternal illness, gestational age, and other factors. For example, the risk of fetal death in a pregnant woman with influenza virus infection requiring admission to hospital was $4 \cdot 2$ (95\% CI 1.4-12.4), ${ }^{20}$ whereas the risk of stillbirth in pregnant women with a mild influenza virus infection was $1 \cdot 9(1 \cdot 1-3 \cdot 4) .{ }^{21}$ The risk of stillbirth in women with HIV infection was 1.67 $(1 \cdot 0-2 \cdot 6) .^{22}$

Although many viral infections increase the risk of fetal death, the mechanism underlying this association is not clear. The analyses of the length of time between symptom onset and stillbirth and of disease severity showed that the risk was highest during acute maternal illness and in women with severe disease, suggesting that the mechanisms involved in the association between dengue infection and stillbirth might be through maternal illness; if the mother becomes severely ill, the fetus could die in the days after symptom onset because of a high fever or other systemic manifestations in the mother. ${ }^{2}$ After this first period, in a small proportion of cases, other mechanisms could be relevant. The proposed mechanism for the effect of some viral infections on pregnancy outcomes includes direct fetus infection leading to damage to vital organs, such as the brain and heart. However, vertical transmission of dengue is not thought to be common, although the virus and antibodies against the virus have been found in placentas, in the cord blood of infants, and in the lung and kidney cells of an aborted fetus. ${ }^{23-28}$ In the absence of vertical transmission, the fetus might be harmed by alterations that remain after the infection is over, such as pathological changes in the placenta that lead to hypoxia, as proposed by Ribeiro and colleagues. ${ }^{29}$ However, we could not elucidate this mechanism in this study, which could be clarified by further research with detailed clinical information (including information about medication and comorbidities) and full investigation by use of appropriate instruments and specific laboratory techniques. These studies could be done with a much smaller number of patients than was used here.

According to a systematic review ${ }^{11}$ of studies of small sample size, ${ }^{11}$ a comment by Carles, ${ }^{30}$ and a study of 
similar Brazilian data, ${ }^{31}$ dengue infection during pregnancy is associated with adverse fetal outcomes, and severe forms of the disease carry a higher risk than less severe forms. This finding was supported by our study, in which the risk of stillbirth among women who had severe forms of dengue was almost three times that of those with mild disease. Because a history of fetal loss is a risk factor for stillbirth, ${ }^{5}$ we investigated whether this variable acted as an effect modifier, but we found that the association was similar for women with and without a previous stillbirth.

Our study had limitations and strengths inherent to the linkage process and to use of secondary data. The main strength of our study was the very large sample size, including all confirmed cases of notified dengue in a country where dengue incidence is high and variable over time. We also had a rigorously selected group of controls and were able to control for confounding. The sensitivity analyses showed the robustness of our findings.

With regard to the use of secondary data, we showed very good agreement on the estimates of risk in cases with and without laboratory confirmation. Our dataset presented some challenges for linkage, and the process and validation of the linkage is discussed in a separate paper. ${ }^{32}$ However, it is unlikely that the linkage process introduced bias, because linkage errors occurred randomly across cases and controls. Missing data were more common in cases than in controls, which might have resulted in better linkage among controls than cases, decreasing the magnitude of the observed association. However, the validation study showed a linkage sensitivity of nearly $60 \%$ for both cases and controls, so the magnitude of the association should not have been affected by linkage error, although the PAF would have been underestimated.

Despite these limitations, we provide evidence that symptomatic dengue infection during pregnancy is a risk factor for stillbirth, with increased risk in severe forms during the acute phase. We recommend further research in different settings to confirm our results and to explore other negative outcomes of pregnancy (preterm birth, intrauterine growth restriction, congenital anomalies, and maternal mortality) and studies of other vector-borne diseases contributing to fetal death. Additional research is required to measure the burden of subclinical maternal viral infections in stillbirth and to elucidate the pathological mechanisms involved in this association. If the association between dengue infection and stillbirth is shown in other studies, recommendations should be made to closely monitor pregnant women with dengue symptoms, to incorporate dengue control into programmes to reduce fetal mortality, and to include pregnant women as an at-risk population in dengue control programmes, to strengthen the health education actions for individual protection of pregnant women.

\section{Contributors}

ESP, MGT, and LCR designed the study. ESP, MdCNC, KH, MFdA, and MLB contributed to the analysis and interpretation. All authors revised the manuscript and approved the final version.

\section{Declaration of interests}

We declare no competing interests.

\section{Acknowledgments}

We thank Robert Picard who wrote the Stata code to match the cases to controls.

\section{References}

1 Lawn JE, Blencowe H, Pattinson R, et al, for The Lancet's Stillbirths Series steering committee. Stillbirths: Where? When? Why? How to make the data count? Lancet 2011; 377: 1448-63.

2 Goldenberg RL, McClure EM, Saleem S, Reddy UM. Infection-related stillbirths. Lancet 2010; 375: 1482-90.

3 Fretts RC, Boyd ME, Usher RH, Usher HA. The changing pattern of fetal death, 1961-1988. Obstet Gynecol 1992; 79: 35-39.

4 Gibbs RS. The origins of stillbirth: infectious diseases. Semin Perinatol 2002; 26: 75-78.

5 Flenady V, Koopmans L, Middleton P, et al. Major risk factors for stillbirth in high-income countries: a systematic review and meta-analysis. Lancet 2011; 377: 1331-40.

6 Williams EJ, Embleton ND, Clark JE, Bythell M, Platt MPW, Berrington JE. Viral infections: contributions to late fetal death stillbirth, and infant death. J Pediatr 2013; 163: 424-28.

7 Craven C, Ward K. Stillbirth: tissue findings with environmental and genetic links. Semin Perinatol 2002; 26: 36-41.

8 Rasmussen SA, Jamienson DJ, Honein MA, Petersen LR. Zika virus and birth defects-reviewing the evidence for causality. N Engl J Med 2016; 374: 1981-87.

9 McClure EM, Dudley DJ, Reddy U, Goldenberg RL. Infectious causes of stillbirth: a clinical perspective. Clin Obstet Gynecol 2010; 53: 635.

10 Pouliot SH, Xiong X, Harville E, et al. Maternal dengue and pregnancy outcomes: a systematic review. Obstet Gynecol Surv 2010; 65: 107-18.

11 Paixão ES, Teixeira MG, Costa Mda C, Rodrigues LC. Dengue during pregnancy and adverse fetal outcomes: a systematic review and meta-analysis. Lancet Infect Dis 2016; 16: 857-65.

12 Friedman EE, Dallah F, Harville EW, et al. Symptomatic dengue infection during pregnancy and infant outcomes: a retrospective cohort study. PLoS Negl Trop Dis 2014; 8: e3226.

13 WHO. Dengue and severe dengue. http://www.who.int/ mediacentre/factsheets/fs117/en (accessed Feb 13, 2017).

14 Ministério da Saúde. Manual de instruções para o preenchimento da declaração de nascido vivo. 2011. http://www.saude.ms.gov.br/ wp-content/uploads/sites/88/2015/11/inst_dn.pdf (accessed Aug 25, 2016).

15 Pedraza DF. Quality of the information system on live births/SINASC: a critical analysis of published studies. Cien Saude Colet 2012; 17: 2729-37 (in Portuguese).

16 Ministério da Saúde. Manual de vigilância do óbito infantil e fetal e do comitê de prevenção do óbito infantil e fetal. 2009. http://bvsms. saude.gov.br/bvs/publicacoes/manual_obito_infantil_fetal_2ed.pdf (accessed Aug 25, 2016).

17 Ministério da Saúde. Dengue diagnóstico e manejo clínico adulto e criança. 2007. http://bvsms.saude.gov.br/bvs/publicacoes/dengue_ diagnostico_manejo_adulto_crianca_3ed.pdf (accessed April 25, 2017)

18 Fellegi IP, Sunter AB. A theory for record linkage. J Am Stat Assoc 1969; 64: 1183-210.

19 Newson RB. Attributable and unattributable risks and fractions and other scenario comparisons. Stata J 2013; 13: 672-98.

20 Pierce M, Kurinczuk JJ, Spark P, Brocklehurst P, Knight M, for UKOSS. Perinatal outcomes after maternal 2009/H1N1 infection: national cohort study. BMJ 2011; 342: d3214.

21 Håberg SE, Trogstad L, Gunnes N, et al. Risk of fetal death after pandemic influenza virus infection or vaccination. $N$ Engl $J$ Med 2013; 368: 333-40.

22 Wedi CO, Kirtley S, Hopewell S, Corrigan R, Kennedy SH, Hemelaar J. Perinatal outcomes associated with maternal HIV infection: a systematic review and meta-analysis. Lancet HIV 2016 3: e33-48. 
23 Ribeiro CF, Lopes VGS, Brasil P, Coelho J, Muniz AG, Nogueira RMR. Perinatal transmission of dengue: a report of 7 cases. J Pediatr 2013; 163: 1514-16

24 Ventura AK, Ehrenkranz NJ, Rosenthal D. Placental passage of antibodies to dengue virus in persons living in a region of hyperendemic dengue virus infection. J Infect Dis 1975 131 (suppl): S62-68.

25 Chye JK, Lim CT, Ng KB, Lim JM, George R, Lam SK. Vertical transmission of dengue. Clin Infect Dis 1997; 25: 1374-77.

26 Perret C, Chanthavanich P, Pengsaa K, et al. Dengue infection during pregnancy and transplacental antibody transfer in Thai mothers. J Infect 2005; 51: 287-93.

27 Argolo AFLT, Féres VCR, Silveira LA, et al. Prevalence and incidence of dengue virus and antibody placental transfer during late pregnancy in central Brazil. BMC Infect Dis 2013; 13: 254

28 Leite RC, Souza AI, Castanha PM, et al. Dengue infection in pregnancy and transplacental transfer of anti-dengue antibodies in Northeast, Brazil. J Clin Virol 2014; 60: 16-21.
29 Ribeiro CF, Lopes VG, Brasil P, Pires AR, Rohloff R, Nogueira RM. Dengue infection in pregnancy and its impact on the placenta. Int J Infect Dis 2017; 55: 109-112.

30 Carles G. What are the true consequences of dengue during pregnancy? Lancet Infect Dis 2016; 16: 765-66.

31 Nascimento LB, Siqueira CM, Coelho GE, Siqueira JB Jr. Symptomatic dengue infection during pregnancy and livebirth outcomes in Brazil, 2007-13: retrospective observational cohort study. Lancet Infect Dis 2017; published online May 18. http://dx.doi:10.1016/S1473-3099(17)30169-X.

32 Paixão ES, Harron K, Andrade K, et al. Evaluation of record linkage of two large administrative databases in a middle income country: stillbirths and notifications of dengue during pregnancy in Brazil. BMC Med Inform Decis Mak 2017; 17: 108. 